\title{
OPERATION MODES AND SINGULARITIES OF 3-PRS PARALLEL MANIPULATORS WITH DIFFERENT ARRANGEMENTS OF P-JOINTS
}

\author{
Latifah Nurahmi ${ }^{1}$, Stéphane Caro ${ }^{2 *}$, Philippe Wenger ${ }^{2}$ \\ ${ }^{1}$ Ecole Centrale de Nantes, IRCCyN, 1, rue de la Noë, 44300 Nantes, France \\ 2 CNRS, IRCCyN, UMR CNRS n 6597, 1, rue de la Noë, 44300 Nantes, France \\ Emails: \{latifah.nurahmi, stephane.caro, philippe.wenger\}@irccyn.ec-nantes.fr
}

\begin{abstract}
The subject of this paper is about the study of the operation modes and the singularity conditions of the 3-PRS parallel manipulator with different arrangements of prismatic joints. The three prismatic joints of the PRS legs are attached to the base with an angle $\alpha$ between the horizontal plane of the base and their directions.

By using an algebraic approach, namely the Study kinematic mapping of the Euclidean group $\mathbf{S E}(\mathbf{3})$, the mechanisms are described by a set of eight constraint equations. A primary decomposition is computed over a set of eight constraint equations and reveals that the 3-PRS manipulators with different arrangements of prismatic joints have identical operation modes, namely $x_{0}=0$ and $x_{3}=0$. Both operation modes are analysed.

The singularity conditions are obtained by deriving the determinant of the Jacobian matrix of the eight constraint equations. All the singular configurations are mapped onto the joint space and are geometrically interpreted. The singularity loci of the 3-PRS parallel manipulators are also traced in its orientation workspace for different values of angle $\alpha$.
\end{abstract}

\section{INTRODUCTION}

Since Hunt proposed the 3-RPS (R, P, S represent revolute, prismatic and spherical joints, respectively) parallel manipulator in 1983 [1], various parallel manipulators which have identical motions have been extensively developed, for example DS Technologie in Germany has developed a machining tool head named

\footnotetext{
*Address all correspondence to this author.
}

the Sprint Z3 [2]. The design of the Sprint Z3 is based on the 3-PRS mechanism in which the prismatic joints are assembled parallel to each other and are vertical. This mechanism is named 3- $\mathrm{P}_{\mathrm{V}} \mathrm{RS}$ by Liu and Bonev in [3], where the subscript $\mathrm{V}$ indicates that the directions of the three prismatic joints are vertical.

In [3], Liu and Bonev showed that the accuracy of the 3$\mathrm{P}_{\mathrm{V}} \mathrm{RS}$ becomes poorer with the increment of the tilt angle of the moving platform. The optimal kinematic design of this mechanism was presented in [4], by introducing three indices that can evaluate the effectiveness of the motion/force transmission. By using these indices, the link lengths were optimized for the purpose of high orientation capability and good motion/force transmissibility.

Tsai et al. in [5] found 64 solutions for the direct kinematics problem by using the Bezout's elimination method to solve three nonlinear trigonometric equations. Fan et al. in [6] analysed this mechanism for machining purposes. The authors also showed that the positioning accuracy of the prismatic joints and the tool length are the most critical parameters that affect the position and the orientation of the spindle platform or the cutter location.

Although the parasitic motion are generally small, they may yield the control of the machine complex and reduce its accuracy. In [7], the authors analysed and compared the parasitic motions of the 3-PVRS mechanism by classifying the geometrical arrangements of the legs and the location of the three spherical joints. Based on this result, Xie et al. in [8] proposed a new architecture of a three degree-of-freedom 3-dof mechanism and analysed its kinematic optimization. In [9], Carbonari et al. proposed a mechatronic system that cooperates two mechanisms of 
3-dof. Both mechanism architectures are 3-CPU (C and U represent cylindrical and universal joints, respectively) manipulators in which the universal joints attached to the platform are assembled differently, i.e., a three degrees of freedom translational motion is generated with the first mechanism, whereas a spherical motion is generated with the second one.

Another architecture of parallel manipulator which is based on the 3-PRS mechanism was proposed by Carretero et al. in [10] for a telescope application. This mechanism is referred to as the $3-\mathrm{P}_{\mathrm{H} S} \mathrm{RS}$ since the prismatic joints are horizontal and they are coplanar to the base. Later in [11], the authors showed the existence of the parasitic motion and concluded that the total translational parasitic motion is free of $z$ component. The design parameters were also optimized to reduce the parasitic motions.

In [12], the authors investigated the singularity conditions for both the 3-P $\mathrm{P} R$ and the $3-\mathrm{P}_{\mathrm{H}} \mathrm{RS}$ manipulators. By using the orientation representation, named Tilt-and-Torsion, the degeneracy of the screw system applied on the platform was detected. By using the screw theory, $\mathrm{Li}$ and $\mathrm{Xu}$ in [13] analysed the kinematic behaviour of the 3-PRS mechanism with layout angle variations of the prismatic joints.

Accordingly, this paper focuses on the study of the operation modes and the singularity conditions of the 3-PRS manipulator for different values of angle $\alpha$, which is the angle between the horizontal plane of the base and the prismatic joint directions. By using Study kinematic mapping of the Euclidean group SE(3) and a primary decomposition based on [14-16], it is shown that the 3-PRS parallel manipulators with any value of $\alpha$ have identical operation modes, namely $x_{0}=0$ and $x_{3}=0$. The singularity conditions of the 3-PRS parallel manipulators are examined in this paper by deriving the determinant of their Jacobian matrix with respect to the Study parameters and their orientation capability are analysed.

This paper is organized as follows: The manipulator architecture is described in Section 2. The constraint equations of the manipulators are expressed in Section 3. A set of constraint equations is used to identify the number and types of operation modes in Section 4. In Section 5, the vanishing conditions of the determinant of the Jacobian matrix are presented and the orientation capabilities of the mechanisms under study are analysed in Section 6. Eventually, the conditions of the actuated lengths corresponding to the transition between the operation modes for five different architectures of the 3-PRS parallel manipulators are discussed in Section 7.

\section{MANIPULATOR ARCHITECTURES}

The 3-PRS parallel manipulator shown in Fig. 1, is composed of an equilateral triangle base, an equilateral triangle platform, and three identical legs. Each leg consists of a prismatic joint, a revolute joint, and a spherical joint mounted in series.

The origin $O$ of the fixed frame $\Sigma_{0}$ is located at the circum-

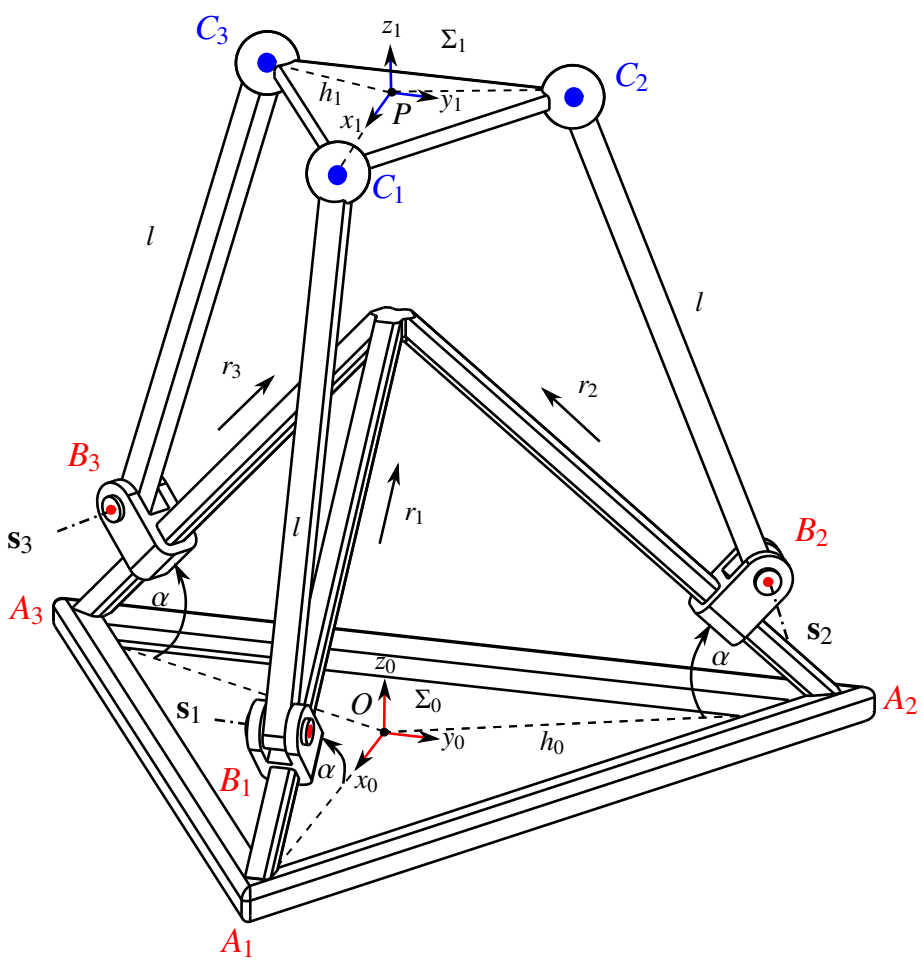

Figure 1: THE 3-PRS PARALLEL MANIPULATOR.

center of the equilateral triangle base. The base is bounded by three vertices $A_{1}, A_{2}, A_{3}$ with circumradius $h_{0}$. The prismatic joint of the $i$-th $(i=1,2,3)$ leg is assembled with an angle $\alpha$ between the horizontal plane of the base and its direction.

The revolute joint of the $i$-th $(i=1,2,3)$ leg is located at point $B_{i}$. Its axis being along vector $\mathbf{s}_{i}$. The origin $P$ of the moving frame $\Sigma_{1}$ is located at the circumcenter of the equilateral triangle platform. The moving platform is bounded by three spherical joints with vertices $C_{1}, C_{2}, C_{3}$ and its circumradius is defined by $h_{1}$.

The segment $B_{i} C_{i}$, of length equal to $l$, is perpendicular to vector $\mathbf{s}_{i}$. As a consequence, the segment $B_{i} C_{i}$ moves in a plane normal to $\mathbf{s}_{i}$. Eventually, the manipulator motions depend on four design parameters $l, h_{0}, h_{1}, \alpha$ and three joint variables $r_{1}, r_{2}, r_{3}$.

\section{CONSTRAINT EQUATIONS}

In this section, the constraint equations are expressed. In the following, $\alpha$ is kept general and we use projective coordinates to define the position vectors of points $B_{i}$ and $C_{i}$. The coordinates of points $B_{i}$ and points $C_{i}$ expressed in $\Sigma_{0}$ and $\Sigma_{1}$ are, respectively: 


$$
\begin{gathered}
\mathbf{r}_{B_{1}}^{0}=\left[1, h_{0}-r_{1} \cos (\alpha), 0, r_{1} \sin (\alpha)\right]^{T}, \\
\mathbf{r}_{B_{2}}^{0}=\left[1,-\frac{1}{2}\left(h_{0}-r_{2} \cos (\alpha)\right), \frac{\sqrt{3}}{2}\left(h_{0}-r_{2} \cos (\alpha)\right), r_{2} \sin (\alpha)\right]^{T}, \\
\mathbf{r}_{B_{3}}^{0}=\left[1,-\frac{1}{2}\left(h_{0}-r_{3} \cos (\alpha)\right),-\frac{\sqrt{3}}{2}\left(h_{0}-r_{3} \cos (\alpha)\right), r_{3} \sin (\alpha)\right]^{T}, \\
\mathbf{r}_{C_{1}}^{1}=\left[1, h_{1}, 0,0\right]^{T}, \\
\mathbf{r}_{C_{2}}^{1}=\left[1,-\frac{1}{2} h_{1}, \frac{\sqrt{3}}{2} h_{1}, 0\right]^{T}, \\
\mathbf{r}_{C_{3}}^{1}=\left[1,-\frac{1}{2} h_{1},-\frac{\sqrt{3}}{2} h_{1}, 0\right]^{T} .
\end{gathered}
$$

To obtain the coordinates of points $C_{1}, C_{2}, C_{3}$ expressed in $\Sigma_{0}$, the Study parametrization of a spatial Euclidean transformation matrix $\mathbf{M} \in \operatorname{SE}(3)$ is used as follows:

$$
\mathbf{M}=\left(\begin{array}{cc}
x_{0}^{2}+x_{1}^{2}+x_{2}^{2}+x_{3}^{2} & \mathbf{0}_{3 \times 1}^{T} \\
\mathbf{M}_{T} & \mathbf{M}_{R}
\end{array}\right)
$$

where $\mathbf{M}_{T}$ and $\mathbf{M}_{R}$ represent the translational and rotational parts of the transformation matrix $\mathbf{M}$, respectively and take the form:

$$
\begin{gathered}
\mathbf{M}_{T}=\left(\begin{array}{c}
2\left(-x_{0} y_{1}+x_{1} y_{0}-x_{2} y_{3}+x_{3} y_{2}\right) \\
2\left(-x_{0} y_{2}+x_{1} y_{3}+x_{2} y_{0}-x_{3} y_{1}\right) \\
2\left(-x_{0} y_{3}-x_{1} y_{2}+x_{2} y_{1}+x_{3} y_{0}\right)
\end{array}\right), \\
\mathbf{M}_{R}=\left(\begin{array}{ccc}
x_{0}^{2}+x_{1}^{2}-x_{2}^{2}-x_{3}^{2} & 2\left(x_{1} x_{2}-x_{0} x_{3}\right) & 2\left(x_{1} x_{3}+x_{0} x_{2}\right) \\
2\left(x_{1} x_{2}+x_{0} x_{3}\right) & x_{0}^{2}-x_{1}^{2}+x_{2}^{2}-x_{3}^{2} & 2\left(x_{2} x_{3}-x_{0} x_{1}\right) \\
2\left(x_{1} x_{3}-x_{0} x_{2}\right) & 2\left(x_{2} x_{3}+x_{0} x_{1}\right) & x_{0}^{2}-x_{1}^{2}-x_{2}^{2}+x_{3}^{2}
\end{array}\right)
\end{gathered}
$$

The parameters $x_{0}, x_{1}, x_{2}, x_{3}, y_{0}, y_{1}, y_{2}, y_{3}$, which appear in matrix M, are called Study-parameters. These parameters make it possible to parametrize $S E(3)$ with dual quaternions. The Study kinematic mapping maps each spatial Euclidean displacement of $S E(3)$ via transformation matrix $\mathbf{M}$ onto a projective point $P\left[x_{0}: x_{1}: x_{2}: x_{3}: y_{0}: y_{1}: y_{2}: y_{3}\right]$ in the 6-dimensional Study quadric $S \in \mathbb{P}^{7}[14]$, such that:

$$
\begin{gathered}
S E(3) \rightarrow P \in \mathbb{P}^{7} \\
\left(x_{0}: x_{1}: x_{2}: x_{3}: y_{0}: y_{1}: y_{2}: y_{3}\right)^{T} \neq(0: 0: 0: 0: 0: 0: 0: 0)^{T}
\end{gathered}
$$

Every projective point $P$ will represent a spatial Euclidean displacement, if it fulfils the following equation and inequality:

$$
x_{0} y_{0}+x_{1} y_{1}+x_{2} y_{2}+x_{3} y_{3}=0, \quad x_{0}^{2}+x_{1}^{2}+x_{2}^{2}+x_{3}^{2} \neq 0
$$

These two conditions will be used in the following computations to simplify the algebraic expressions. First of all, the halftangent substitutions for $\alpha$ are performed to remove the trigonometric functions:

$$
\cos (\alpha)=\frac{1-t_{a}^{2}}{1+t_{a}^{2}}, \quad \sin (\alpha)=\frac{2 t_{a}}{1+t_{a}^{2}}
$$

where $t_{a}=\tan \left(\frac{\alpha}{2}\right)$. The coordinates of points $C_{i}$ expressed in $\Sigma_{0}$ are obtained by:

$$
\mathbf{r}_{C_{i}}^{0}=\mathbf{M} \mathbf{r}_{C_{i}}^{1} \quad i=0, \ldots, 3
$$

As the coordinates of all points are given in terms of Study parameters, the design parameters and the joint variables; the constraint equations can be obtained by examining the design of the manipulators. The segment connecting points $B_{i}$ and $C_{i}$ is orthogonal to the axis $\mathbf{s}_{i}$ of the $i$-th revolute joint, with:

$$
\mathbf{s}_{1}=\left[\begin{array}{l}
0 \\
0 \\
1 \\
0
\end{array}\right], \quad \mathbf{s}_{2}=\left[\begin{array}{c}
0 \\
-\frac{\sqrt{3}}{2} \\
-\frac{1}{2} \\
0
\end{array}\right], \quad \mathbf{s}_{3}=\left[\begin{array}{c}
0 \\
\frac{\sqrt{3}}{2} \\
-\frac{1}{2} \\
0
\end{array}\right]
$$

Accordingly, the scalar product of vector $\left(\mathbf{r}_{C_{i}}^{0}-\mathbf{r}_{B_{i}}^{0}\right)$ and vector $\mathbf{s}_{i}$ vanishes, namely:

$$
\left(\mathbf{r}_{C_{i}}^{0}-\mathbf{r}_{B_{i}}^{0}\right)^{T} \mathbf{s}_{i}=0
$$

After computing the corresponding scalar products and removing the common denominators $\left(x_{0}^{2}+x_{1}^{2}+x_{2}^{2}+x_{3}^{2}\right)$, the following three equations come out:

$$
\begin{aligned}
& g_{1}: x_{0} x_{3}=0 \\
& g_{2}: 2 h_{1} x_{0} x_{3}-h_{1} x_{1} x_{2}+x_{0} y_{2}-x_{1} y_{3}-x_{2} y_{0}+x_{3} y_{1}=0 \\
& g_{3}: h_{1} x_{1}^{2}-h_{1} x_{2}^{2}+2 x_{0} y_{1}-2 x_{1} y_{0}+2 x_{2} y_{3}-2 x_{3} y_{2}=0
\end{aligned}
$$

Noticeably, $t_{a}$ does not appear in Eq. (10). It means that the three constraint equations in Eq. (10) are valid for all the 3-PRS parallel manipulators with any value of parameter angle $\alpha$. 
To derive the constraint equations corresponding to the segment $B_{i} C_{i}$, the design parameter $l$ is given. It follows that point $C_{i}$ has the freedom to move along a circle of center $B_{i}$ and the distance equation can be formulated as $\left\|\left(\mathbf{r}_{C_{i}}^{0}-\mathbf{r}_{B_{i}}^{0}\right)\right\|^{2}=l^{2}$. As a consequence, the following three equations are obtained:

$g_{4}:\left(h_{0}^{2} t_{a}^{2}-2 h_{0} h_{1} t_{a}^{2}+2 h_{0} r_{1} t_{a}^{2}+h_{1}^{2} t_{a}^{2}-2 h_{1} r_{1} t_{a}^{2}-l^{2} t_{a}^{2}+r_{1}^{2} t_{a}^{2}+\right.$ $\left.h_{0}^{2}-2 h_{0} h_{1}-2 h_{0} r_{1}+h_{1}^{2}+2 h_{1} r_{1}-l^{2}+r_{1}^{2}\right) x_{0}^{2}+8 t_{a} h_{1} r_{1} x_{0} x_{2}$ $+\left(4 h_{0} t_{a}^{2}-4 h_{1} t_{a}^{2}+4 r_{1} t_{a}^{2}+4 h_{0}-4 h_{1}-4 r_{1}\right) x_{0} y_{1}+8 t_{a} r_{1} x_{0}$ $y_{3}+\left(h_{0}^{2} t_{a}^{2}-2 h_{0} h_{1} t_{a}^{2}+2 h_{0} r_{1} t_{a}^{2}+h_{1}^{2} t_{a}^{2}-2 h_{1} r_{1} t_{a}^{2}-l^{2} t_{a}^{2}+r_{1}^{2}\right.$ $\left.t_{a}^{2}+h_{0}^{2}-2 h_{0} h_{1}-2 h_{0} r_{1}+h_{1}^{2}+2 h_{1} r_{1}-l^{2}+r_{1}^{2}\right) x_{1}^{2}-8 t_{a} h_{1} r_{1}$ $x_{1} x_{3}+\left(-4 h_{0} t_{a}^{2}+4 h_{1} t_{a}^{2}-4 r_{1} t_{a}^{2}-4 h_{0}+4 h_{1}+4 r_{1}\right) x_{1} y_{0}+8$ $t_{a} r_{1} x_{1} y_{2}+\left(h_{0}^{2} t_{a}^{2}+2 h_{0} h_{1} t_{a}^{2}+2 h_{0} r_{1} t_{a}^{2}+h_{1}^{2} t_{a}^{2}+2 h_{1} r_{1} t_{a}^{2}-l^{2}\right.$ $\left.t_{a}^{2}+r_{1}^{2} t_{a}^{2}+h_{0}^{2}+2 h_{0} h_{1}-2 h_{0} r_{1}+h_{1}^{2}-2 h_{1} r_{1}-l^{2}+r_{1}^{2}\right) x_{2}^{2}-8$ $t_{a} r_{1} x_{2} y_{1}+\left(4 h_{0} t_{a}^{2}+4 h_{1} t_{a}^{2}+4 r_{1} t_{a}^{2}+4 h_{0}+4 h_{1}-4 r_{1}\right) x_{2} y_{3}+$ $\left(h_{0}^{2} t_{a}^{2}+2 h_{0} h_{1} t_{a}^{2}+2 h_{0} r_{1} t_{a}^{2}+h_{1}^{2} t_{a}^{2}+2 h_{1} r_{1} t_{a}^{2}-l^{2} t_{a}^{2}+r_{1}^{2} t_{a}^{2}+\right.$ $\left.h_{0}^{2}+2 h_{0} h_{1}-2 h_{0} r_{1}+h_{1}^{2}-2 h_{1} r_{1}-l^{2}+r_{1}^{2}\right) x_{3}^{2}-8 t_{a} r_{1} x_{3} y_{0}+$ $\left(-4 h_{0} t_{a}^{2}-4 h_{1} t_{a}^{2}-4 r_{1} t_{a}^{2}-4 h_{0}-4 h_{1}+4 r_{1}\right) x_{3} y_{2}+\left(4 t_{a}^{2}+4\right)$ $\left(y_{0}^{2}+y_{1}^{2}+y_{2}^{2}+y_{3}^{2}\right)=0$

$g_{5}:\left(2 \sqrt{3} h_{0} t_{a}^{2}+2 \sqrt{3} h_{1} t_{a}^{2}+2 \sqrt{3} r_{2} t_{a}^{2}+2 \sqrt{3} h_{0}+2 \sqrt{3} h_{1}-2 \sqrt{3} r_{2}\right)$ $x_{3} y_{1}+\left(-2 \sqrt{3} h_{0} t_{a}^{2}+2 \sqrt{3} h_{1} t_{a}^{2}-2 \sqrt{3} r_{2} t_{a}^{2}-2 \sqrt{3} h_{0}+2 \sqrt{3} h_{1}\right.$ $\left.+2 \sqrt{3} r_{2}\right) x_{2} y_{0}+4 t_{a} h_{1} r_{2} x_{1} x_{3}+\left(-2 \sqrt{3} h_{0} t_{a}^{2}-2 \sqrt{3} h_{1} t_{a}^{2}-2\right.$ $\left.\sqrt{3} r_{2} t_{a}^{2}-2 \sqrt{3} h_{0}-2 \sqrt{3} h_{1}+2 \sqrt{3} r_{2}\right) x_{1} y_{3}+\left(2 \sqrt{3} h_{0} t_{a}^{2}-2 \sqrt{3}\right.$ $\left.h_{1} t_{a}^{2}+2 \sqrt{3} r_{2} t_{a}^{2}+2 \sqrt{3} h_{0}-2 \sqrt{3} h_{1}-2 \sqrt{3} r_{2}\right) x_{0} y_{2}+\left(2 \sqrt{3} h_{0} h_{1}\right.$ $\left.t_{a}^{2}+2 \sqrt{3} h_{1} r_{2} t_{a}^{2}+2 \sqrt{3} h_{0} h_{1}-2 \sqrt{3} h_{1} r_{2}\right) x_{1} x_{2}+\left(-2 h_{0} t_{a}^{2}+2 h_{1}\right.$ $\left.t_{a}^{2}-2 r_{2} t_{a}^{2}-2 h_{0}+2 h_{1}+2 r_{2}\right) x_{0} y_{1}+\left(2 h_{0} t_{a}^{2}-2 h_{1} t_{a}^{2}+2 r_{2} t_{a}^{2}+\right.$ $\left.2 h_{0}-2 h_{1}-2 r_{2}\right) x_{1} y_{0}+\left(-2 h_{0} t_{a}^{2}-2 h_{1} t_{a}^{2}-2 r_{2} t_{a}^{2}-2 h_{0}-2 h_{1}\right.$ $\left.+2 r_{2}\right) x_{2} y_{3}+\left(2 h_{0} t_{a}^{2}+2 h_{1} t_{a}^{2}+2 r_{2} t_{a}^{2}+2 h_{0}+2 h_{1}-2 r_{2}\right) x_{3} y_{2}$ $-4 t_{a} h_{1} r_{2} x_{0} x_{2}+\left(4 t_{a}^{2}+4\right) y_{0}^{2}+\left(4 t_{a}^{2}+4\right) y_{2}^{2}+\left(4 t_{a}^{2}+4\right) y_{1}^{2}+(4$ $\left.t_{a}^{2}+4\right) y_{3}^{2}-4 t_{a} \sqrt{3} h_{1} r_{2} x_{2} x_{3}-4 t_{a} \sqrt{3} h_{1} r_{2} x_{0} x_{1}+\left(h_{0}^{2} t_{a}^{2}-2 h_{0}\right.$ $h_{1} t_{a}^{2}+2 h_{0} r_{2} t_{a}^{2}+h_{1}^{2} t_{a}^{2}-2 h_{1} r_{2} t_{a}^{2}-l^{2} t_{a}^{2}+r_{2}^{2} t_{a}^{2}+h_{0}^{2}-2 h_{0} h_{1}-$ $\left.2 h_{0} r_{2}+h_{1}^{2}+2 h_{1} r_{2}-l^{2}+r_{2}^{2}\right) x_{0}^{2}+\left(h_{0}^{2} t_{a}^{2}+h_{0} h_{1} t_{a}^{2}+2 h_{0} r_{2} t_{a}^{2}+\right.$ $h_{1}^{2} t_{a}^{2}+h_{1} r_{2} t_{a}^{2}-l^{2} t_{a}^{2}+r_{2}^{2} t_{a}^{2}+h_{0}^{2}+h_{0} h_{1}-2 h_{0} r_{2}+h_{1}^{2}-h_{1} r_{2}-$ $\left.l^{2}+r_{2}^{2}\right) x_{1}^{2}+\left(h_{0}^{2} t_{a}^{2}-h_{0} h_{1} t_{a}^{2}+2 h_{0} r_{2} t_{a}^{2}+h_{1}^{2} t_{a}^{2}-h_{1} r_{2} t_{a}^{2}-l^{2} t_{a}^{2}\right.$ $\left.+r_{2}^{2} t_{a}^{2}+h_{0}^{2}-h_{0} h_{1}-2 h_{0} r_{2}+h_{1}^{2}+h_{1} r_{2}-l^{2}+r_{2}^{2}\right) x_{2}^{2}+\left(h_{0}^{2} t_{a}^{2}+\right.$ $2 h_{0} h_{1} t_{a}^{2}+2 h_{0} r_{2} t_{a}^{2}+h_{1}^{2} t_{a}^{2}+2 h_{1} r_{2} t_{a}^{2}-l^{2} t_{a}^{2}+r_{2}^{2} t_{a}^{2}+h_{0}^{2}+2 h_{0} h_{1}$ $\left.-2 h_{0} r_{2}+h_{1}^{2}-2 h_{1} r_{2}-l^{2}+r_{2}^{2}\right) x_{3}^{2}+8 t_{a} r_{2} x_{0} y_{3}+8 t_{a} r_{2} x_{1} y_{2}-$
$8 t_{a} r_{2} x_{2} y_{1}-8 t_{a} r_{2} x_{3} y_{0}=0$

$g_{6}: 4 t_{a} h_{1} r_{3} x_{1} x_{3}-4 t_{a} h_{1} r_{3} x_{0} x_{2}+8 t_{a} r_{3} x_{0} y_{3}+8 t_{a} r_{3} x_{1} y_{2}-8 t_{a} r_{3}$ $x_{2} y_{1}-8 t_{a} r_{3} x_{3} y_{0}+\left(2 h_{0} t_{a}^{2}+2 h_{1} t_{a}^{2}+2 r_{3} t_{a}^{2}+2 h_{0}+2 h_{1}-2 r_{3}\right)$ $x_{3} y_{2}+\left(-2 h_{0} t_{a}^{2}+2 h_{1} t_{a}^{2}-2 r_{3} t_{a}^{2}-2 h_{0}+2 h_{1}+2 r_{3}\right) x_{0} y_{1}+\left(2 h_{0}\right.$ $\left.t_{a}^{2}-2 h_{1} t_{a}^{2}+2 r_{3} t_{a}^{2}+2 h_{0}-2 h_{1}-2 r_{3}\right) x_{1} y_{0}+\left(-2 h_{0} t_{a}^{2}-2 h_{1} t_{a}^{2}\right.$ $\left.-2 r_{3} t_{a}^{2}-2 h_{0}-2 h_{1}+2 r_{3}\right) x_{2} y_{3}+\left(4 t_{a}^{2}+4\right) y_{0}^{2}+\left(4 t_{a}^{2}+4\right) y_{2}^{2}+$ $\left(4 t_{a}^{2}+4\right) y_{1}^{2}+\left(4 t_{a}^{2}+4\right) y_{3}^{2}+\left(h_{0}^{2} t_{a}^{2}-2 h_{0} h_{1} t_{a}^{2}+2 h_{0} r_{3} t_{a}^{2}+h_{1}^{2} t_{a}^{2}\right.$ $-2 h_{1} r_{3} t_{a}^{2}-l^{2} t_{a}^{2}+r_{3}^{2} t_{a}^{2}+h_{0}^{2}-2 h_{0} h_{1}-2 h_{0} r_{3}+h_{1}^{2}+2 h_{1} r_{3}-$ $\left.l^{2}+r_{3}^{2}\right) x_{0}^{2}+\left(h_{0}^{2} t_{a}^{2}+h_{0} h_{1} t_{a}^{2}+2 h_{0} r_{3} t_{a}^{2}+h_{1}^{2} t_{a}^{2}+h_{1} r_{3} t_{a}^{2}-l^{2} t_{a}^{2}\right.$ $\left.+r_{3}^{2} t_{a}^{2}+h_{0}^{2}+h_{0} h_{1}-2 h_{0} r_{3}+h_{1}^{2}-h_{1} r_{3}-l^{2}+r_{3}^{2}\right) x_{1}^{2}+\left(h_{0}^{2} t_{a}^{2}-\right.$ $h_{0} h_{1} t_{a}^{2}+2 h_{0} r_{3} t_{a}^{2}+h_{1}^{2} t_{a}^{2}-h_{1} r_{3} t_{a}^{2}-l^{2} t_{a}^{2}+r_{3}^{2} t_{a}^{2}+h_{0}^{2}-h_{0} h_{1}-$ $\left.2 h_{0} r_{3}+h_{1}^{2}+h_{1} r_{3}-l^{2}+r_{3}^{2}\right) x_{2}^{2}+\left(h_{0}^{2} t_{a}^{2}+2 h_{0} h_{1} t_{a}^{2}+2 h_{0} r_{3} t_{a}^{2}+\right.$ $h_{1}^{2} t_{a}^{2}+2 h_{1} r_{3} t_{a}^{2}-l^{2} t_{a}^{2}+r_{3}^{2} t_{a}^{2}+h_{0}^{2}+2 h_{0} h_{1}-2 h_{0} r_{3}+h_{1}^{2}-2 h_{1}$ $\left.r_{3}-l^{2}+r_{3}^{2}\right) x_{3}^{2}+\left(2 \sqrt{3} h_{0} t_{a}^{2}-2 \sqrt{3} h_{1} t_{a}^{2}+2 \sqrt{3} r_{3} t_{a}^{2}+2 \sqrt{3} h_{0}-\right.$ $\left.2 \sqrt{3} h_{1}-2 \sqrt{3} r_{3}\right) x_{2} y_{0}+\left(2 \sqrt{3} h_{0} t_{a}^{2}+2 \sqrt{3} h_{1} t_{a}^{2}+2 \sqrt{3} r_{3} t_{a}^{2}+2\right.$ $\left.\sqrt{3} h_{0}+2 \sqrt{3} h_{1}-2 \sqrt{3} r_{3}\right) x_{1} y_{3}+\left(-2 \sqrt{3} h_{0} h_{1} t_{a}^{2}-2 \sqrt{3} h_{1} r_{3} t_{a}^{2}\right.$ $\left.-2 \sqrt{3} h_{0} h_{1}+2 \sqrt{3} h_{1} r_{3}\right) x_{1} x_{2}+\left(-2 \sqrt{3} h_{0} t_{a}^{2}+2 \sqrt{3} h_{1} t_{a}^{2}-2 \sqrt{3}\right.$ $\left.r_{3} t_{a}^{2}-2 \sqrt{3} h_{0}+2 \sqrt{3} h_{1}+2 \sqrt{3} r_{3}\right) x_{0} y_{2}+\left(-2 \sqrt{3} h_{0} t_{a}^{2}-2 \sqrt{3} h_{1}\right.$ $\left.t_{a}^{2}-2 \sqrt{3} r_{3} t_{a}^{2}-2 \sqrt{3} h_{0}-2 \sqrt{3} h_{1}+2 \sqrt{3} r_{3}\right) x_{3} y_{1}+4 \sqrt{3} t_{a} h_{1} r_{3}$ $x_{0} x_{1}+4 \sqrt{3} t_{a} h_{1} r_{3} x_{2} x_{3}=0$

The Study-equation in Eq. (5) is added since all solutions have to be within the Study-quadric, i.e.:

$$
g_{7}: x_{0} y_{0}+x_{1} y_{1}+x_{2} y_{2}+x_{3} y_{3}=0
$$

Under the condition $\left(x_{0}^{2}+x_{1}^{2}+x_{2}^{2}+x_{3}^{2} \neq 0\right)$, we can find all possible points in $\mathbb{P}^{7}$ that fulfil these seven equations. To exclude the exceptional generator $\left(x_{0}=x_{1}=x_{2}=x_{3}=0\right)$, we add the following normalization equation:

$$
g_{8}: x_{0}^{2}+x_{1}^{2}+x_{2}^{2}+x_{3}^{2}-1=0
$$

It assures that there is no point of the exceptional generator appears as a solution. However, for each projective solution point, we obtain two affine representatives. This has to be taken into account for the enumeration of the number of solutions. 


\section{OPERATION MODES}

The set of eight constraint equations is always written as polynomial ideal with variables $\left\{x_{0}, x_{1}, x_{2}, x_{3}, y_{0}, y_{1}, y_{2}, y_{3}\right\}$ over the coefficient ring $\mathbb{C}\left[h_{0}, h_{1}, l, t_{a}, r_{1}, r_{2}, r_{3}\right]$, as:

$$
\mathcal{I}=\left\langle g_{1}, g_{2}, g_{3}, g_{4}, g_{5}, g_{6}, g_{7}, g_{8}\right\rangle
$$

At this point, the following ideal which is independent of the joint variables $r_{1}, r_{2}$ and $r_{3}$, is examined:

$$
\mathcal{J}=\left\langle g_{1}, g_{2}, g_{3}, g_{7}\right\rangle
$$

The primary decomposition is computed to verify if the ideal $\mathcal{J}$ is the intersection of several smaller ideals. The primary decomposition returns several $\mathcal{J}_{i}$ in which $\mathcal{J}=\bigcap_{i} \mathcal{J}_{i}$. In other words, the vanishing set is given by $\mathbf{V}(\mathcal{J})=\bigcup_{i} \mathbf{V}\left(\mathcal{J}_{i}\right)$. It expresses that the variety $\mathbf{V}(\mathcal{J})$ is the union of some other or simpler varieties $\mathbf{V}\left(\mathcal{J}_{i}\right)$.

The primary decomposition geometrically tells that the intersection of those equations will split into smaller parts. Indeed, the result shows that the ideal $\mathcal{J}$ is decomposed into three components $\mathcal{J}_{i}$ as:

$$
\mathcal{J}=\bigcap_{i=1}^{3} \mathcal{J}_{i}
$$

with the results of primary decomposition:

$$
\begin{aligned}
\mathcal{J}_{1}: & \left\langle x_{0}, x_{1} y_{1}+x_{2} y_{2}+x_{3} y_{3}, h_{1} x_{1} x_{2}+x_{2} y_{0}-x_{3} y_{1}+x_{1} y_{3},\right. \\
& h_{1} x_{1}^{2}-h_{1} x_{2}^{2}-2 x_{1} y_{0}-2 x_{3} y_{2}+2 x_{2} y_{3}, h_{1} x_{2} y_{1}^{2}-3 h_{1} x_{2} y_{2}^{2} \\
& +h_{1}^{2} x_{2}^{2} y_{3}+h_{1} x_{1} y_{0} y_{3}-2 y_{0}^{2} y_{3}-2 y_{1}^{2} y_{3}-h_{1} x_{3} y_{2} y_{3}-2 \\
& y_{2}^{2} y_{3}-h_{1} x_{2} y_{3}^{2}-2 y_{3}^{3}, x_{2} y_{0} y_{1}-x_{3} y_{1}^{2}-h_{1} x_{2}^{2} y_{2}-h_{1} x_{2} x_{3} \\
& y_{3}+x_{1} y_{1} y_{3}, h_{1} x_{2}^{2} y_{1}+2 x_{1} y_{0} y_{1}+h_{1} x_{1} x_{2} y_{2}+2 x_{3} y_{1} y_{2}+ \\
& h_{1} x_{1} x_{3} y_{3}-2 x_{2} y_{1} y_{3}, h_{1} x_{2}^{3}+3 x_{1} x_{2} y_{0}-x_{1} x_{3} y_{1}+2 x_{2} x_{3} \\
& y_{2}+x_{1}^{2} y_{3}-2 x_{2}^{2} y_{3}, h_{1} x_{3} y_{1}^{3}+h_{1}^{2} x_{2}^{2} y_{1} y_{2}-3 h_{1} x_{2} y_{0} y_{2}^{2}+h_{1}^{2} \\
& x_{2}^{2} y_{0} y_{3}+h_{1} x_{1} y_{0}^{2} y_{3}-2 y_{0}^{3} y_{3}+h_{1}^{2} x_{2} x_{3} y_{1} y_{3}-h_{1} x_{1} y_{1}^{2} y_{3}- \\
& \left.2 y_{0} y_{1}^{2} y_{3}-h_{1} x_{3} y_{0} y_{2} y_{3}-2 y_{0} y_{2}^{2} y_{3}-h_{1} x_{2} y_{0} y_{3}^{2}-2 y_{0} y_{3}^{3}\right\rangle \\
\mathcal{J}_{2}: & \left\langle x_{3}, x_{0} y_{0}+x_{1} y_{1}+x_{2} y_{2}, h_{1} x_{1} x_{2}+x_{2} y_{0}-x_{0} y_{2}+x_{1} y_{3},\right. \\
& h_{1} x_{1}^{2}-h_{1} x_{2}^{2}-2 x_{1} y_{0}+2 x_{0} y_{1}+2 x_{2} y_{3}, h_{1}^{2} x_{2}^{2} y_{0}+h_{1} x_{1} y_{0}^{2} \\
& -2 y_{0}^{3}-2 y_{0} y_{1}^{2}+3 h_{1} x_{2} y_{1} y_{2}+h_{1} x_{1} y_{2}^{2}-2 y_{0} y_{2}^{2}-h_{1} x_{2} y_{0}
\end{aligned}
$$

$$
\begin{aligned}
& y_{3}-2 y_{0} y_{3}^{2}, h_{1} x_{2}^{3}+3 x_{1} x_{2} y_{0}-2 x_{0} x_{2} y_{1}-x_{0} x_{1} y_{2}+x_{1}^{2} y_{3} \\
& \left.-2 x_{2}^{2} y_{3}\right\rangle \\
\mathcal{J}_{3}: & \left\langle x_{0}, x_{1}, x_{2}, x_{3}\right\rangle
\end{aligned}
$$

An inspection of the vanishing set $\mathbf{V}\left(\mathcal{J}_{3} \cup g_{8}\right)$ gives an empty result, since the set of polynomials $\left\{x_{0}, x_{1}, x_{2}, x_{3}, x_{0}^{2}+x_{1}^{2}+x_{2}^{2}+\right.$ $\left.x_{3}^{2}-1=0\right\}$ can never vanish simultaneously over $\mathbb{R}$ or $\mathbb{C}$. Therefore, only two components are left. As a consequence, the 3-PRS manipulators with any value of $\alpha$ have two operation modes, which are characterized by $x_{0}=0$ and $x_{3}=0$. It turns out that these operation modes are also operation modes of the 3-RPS parallel manipulator studied in [15].

To complete the analysis, the remaining equations have to be added by writing:

$$
\mathcal{K}_{i}=\mathcal{J}_{i} \cup\left\langle g_{4}, g_{5}, g_{6}, g_{8}\right\rangle, \quad i=1,2
$$

Each system $\mathcal{K}_{i}$ corresponds to a specific operation mode of the 3-PRS parallel manipulators. In the following, each system $\mathcal{K}_{i}$ will be discussed separately.

\section{System $\mathcal{K}_{1}$ : Operation mode $x_{0}=0$}

In this operation mode, the moving platform is reversed about an axis defined by $\left(x_{1}, x_{2}, x_{3}\right)$ by 180 degrees [17] from the identity condition (when the fixed frame $\Sigma_{0}$ and the moving frame $\Sigma_{1}$ are coincident). The condition $x_{0}=0$ is valid for all poses of the manipulators. The variables $y_{0}, y_{1}, y_{3}$ can be solved linearly from the ideal $\mathcal{J}_{1}$ and they are substituted back into the matrix $\mathbf{M}$, as:

$$
\mathbf{T}_{1}=\left(\begin{array}{cccc}
1 & 0 & 0 & 0 \\
h_{1}\left(x_{1}^{2}-x_{2}^{2}\right) & x_{1}^{2}-x_{2}^{2}-x_{3}^{2} & 2 x_{1} x_{2} & 2 x_{1} x_{3} \\
-2 h_{1} x_{1} x_{2} & 2 x_{1} x_{2} & -\left(x_{1}^{2}-x_{2}^{2}+x_{3}^{2}\right) & 2 x_{2} x_{3} \\
Z & 2 x_{1} x_{3} & 2 x_{2} x_{3} & -\left(x_{1}^{2}+x_{2}^{2}-x_{3}^{2}\right)
\end{array}\right)
$$

where $Z=\frac{h_{1}\left(x_{1}^{2} x_{3}-x_{2}^{2} x_{3}\right)-2\left(x_{1}^{2} y_{2}+x_{2}^{2} y_{2}+x_{3}^{2} y_{2}\right)}{x_{1}}$

From the transformation matrix $\mathbf{T}_{1}$, it can be seen that the translational motions can be parametrized by $y_{2}$ and the rotational motions can be parametrized by $x_{1}, x_{2}, x_{3}$ in connection with $x_{1}^{2}+x_{2}^{2}+x_{3}^{2}-1=0$ [19].

\section{System $\mathcal{K}_{2}$ : Operation mode $x_{3}=0$}

In this operation mode, the moving platform is rotated about and translated along an axis parallel to the xy-plane [17] from 
the identity condition. The rotational angle $\varphi$ of the transformation can be enumerated directly from $\cos \left(\frac{\varphi}{2}\right)=x_{0}$, whereas the translational distance $s$ of the transformation can be computed from the Study parameters [18], as follows:

$$
s=\frac{2 y_{0}}{\sqrt{x_{1}^{2}+x_{2}^{2}+x_{3}^{2}}}
$$

The condition $x_{3}=0$ is valid for all poses of the manipulators and the variables $y_{0}, y_{2}, y_{3}$ can be solved linearly from the ideal $\mathcal{J}_{2}$. All variables are substituted back into the matrix $\mathbf{M}$, as:

$$
\mathbf{T}_{2}=\left(\begin{array}{cccc}
1 & 0 & 0 & 0 \\
h_{1}\left(x_{1}^{2}-x_{2}^{2}\right) & x_{0}^{2}+x_{1}^{2}-x_{2}^{2} & 2 x_{1} x_{2} & 2 x_{0} x_{2} \\
-2 h_{1} x_{1} x_{2} & 2 x_{1} x_{2} & x_{0}^{2}-x_{1}^{2}+x_{2}^{2} & -2 x_{0} x_{1} \\
Z & -2 x_{0} x_{2} & 2 x_{0} x_{1} & x_{0}^{2}-x_{1}^{2}-x_{2}^{2}
\end{array}\right)
$$

where $Z=\frac{h_{1}\left(x_{1}^{2} x_{0}-x_{2}^{2} x_{0}\right)+2\left(x_{0}^{2} y_{1}+x_{1}^{2} y_{1}+x_{2}^{2} y_{1}\right)}{x_{2}}$

It can be seen from the transformation matrix $\mathbf{T}_{2}$ that the translational motions can be parametrized by $y_{1}$ and the rotational motions can be parametrized by $x_{0}, x_{1}, x_{2}$ in connection with $x_{0}^{2}+x_{1}^{2}+x_{2}^{2}-1=0$ [19].

\section{SINGULARITY CONDITIONS}

The 3-PRS parallel manipulators reach a singular configuration when the determinant of its Jacobian matrix vanishes. The Jacobian matrix is the matrix of all first order partial derivatives of eight constraint equations $\left\{g_{1}, g_{2}, g_{3}, g_{4}, g_{5}, g_{6}, g_{7}, g_{8}\right\}$ with respect to the Study parameters $\left\{x_{0}, x_{1}, x_{2}, x_{3}, y_{0}, y_{1}, y_{2}, y_{3}\right\}$.

Since the mechanisms have more than one operation mode, the singular configurations can be classified into two different types, i.e. the configurations that belong to a single operation mode and the configurations that belong to more than one operation mode. The common configurations that belong to more than one operation mode allow the mechanisms to switch from one operation mode to another operation mode.

The singular poses are examined by taking the Jacobian matrix from each system of polynomial $\mathcal{K}_{i}(i=1,2)$ and computing its determinant $\mathcal{S}_{i}: \operatorname{det}\left(\mathbf{J}_{i}\right)=0$. The determinant of each system splits into two factors, as:

$$
\begin{aligned}
& \mathcal{S}_{1}: p^{13}\left(x_{1}, x_{2}, x_{3}, y_{0}, y_{1}, y_{2}, y_{3}, t_{a}, r_{1}, r_{2}, r_{3}\right) \cdot x_{3} \\
& \mathcal{S}_{2}: p^{13}\left(x_{0}, x_{1}, x_{2}, y_{0}, y_{1}, y_{2}, y_{3}, t_{a}, r_{1}, r_{2}, r_{3}\right) \cdot x_{0}
\end{aligned}
$$

The first factors of $\mathcal{S}_{1}$ and $\mathcal{S}_{2}$ in Eq. (24) give the singularity conditions inside the system $\mathcal{K}_{1}$ and the system $\mathcal{K}_{2}$, respectively. Under these singularity conditions, the orientation capability of the 3-PRS manipulator with different value of angle $\alpha$ in each system $\mathcal{K}_{i}$ will be analysed hereafter.

The second factors of each determinant from Eq. (24) lead to the singularity configurations that belong to the system $\mathcal{K}_{1}$ and the system $\mathcal{K}_{2}$ simultaneously, i.e. $x_{0}=x_{3}=0$. These configurations are the transition configurations that allow the 3-PRS parallel manipulators to switch from $\mathcal{K}_{1}$ to $\mathcal{K}_{2}$ and vice versa.

\section{ORIENTATION CAPABILITY ANALYSIS}

In this paper, the Euler angles based on [20] (or $w-u-w$ Euler angles by Tsai [21]) are used to parametrize the orientation of the moving platform. It is possible to represent the rotational parts of the Study parameters in terms of the Euler angles $(\phi, \theta, \psi)$ [22], as follows:

$$
\begin{aligned}
& x_{0}=\cos \left(\frac{\theta}{2}\right) \cos \left(\frac{\phi}{2}+\frac{\psi}{2}\right) \\
& x_{1}=\sin \left(\frac{\theta}{2}\right) \cos \left(\frac{\phi}{2}-\frac{\psi}{2}\right) \\
& x_{2}=\sin \left(\frac{\theta}{2}\right) \sin \left(\frac{\phi}{2}-\frac{\psi}{2}\right) \\
& x_{3}=\cos \left(\frac{\theta}{2}\right) \sin \left(\frac{\phi}{2}+\frac{\psi}{2}\right)
\end{aligned}
$$

An orientation capability of the 3-PRS parallel manipulators based on $[3,4]$ is defined as the maximum tilt angle $\theta$ for a given azimuth angle $\phi$. The maximum orientation capability of the moving platform depends mostly on the singularities which are derived from the first factors of the determinant of the Jacobian matrix in Eq. (24). The second factors are not considered since the corresponding singularities occur on the boundary of each operation mode, namely when the platform is tilted by $\theta=\pi$. These singularities appear at the transition between the two operation modes.

\section{Orientation Capability in Operation Mode $x_{0}=0$}

In this operation mode, the variables $y_{0}, y_{1}, y_{3}$ are solved linearly from the ideal $\mathcal{J}_{1}$. The prismatic lengths $r_{1}, r_{2}, r_{3}$ are obtained from the equations $g_{4}, g_{5}, g_{6}$ in Eqs. (11-12), respectively. All joint variables and the Study parameters are substituted into the first factor of the determinant of the Jacobian matrix $\mathcal{S}_{1}$ : $\operatorname{det}\left(\mathbf{J}_{1}\right)=0$, hence it is determined in terms of $t_{a}, x_{1}, x_{2}, x_{3}, y_{2}$.

To obtain the orientation of the moving platform, the remaining Study parameters are converted into the tilt and azimuth angles in Eq. (26) and the corresponding transformation matrix $\mathbf{M}_{1}$ is shown in Eq. (27). The altitude of the moving platform $Z$ can be determined as function of parameter $y_{2}$ and tilt and azimuth 
angles. The design parameters are assigned as $h_{0}=2 \mathrm{~m}, h_{1}=1 \mathrm{~m}$, $l=5 \mathrm{~m}$.

$$
x_{1}=\sin \left(\frac{\theta}{2}\right) \sin (\phi), \quad x_{2}=-\sin \left(\frac{\theta}{2}\right) \cos (\phi), \quad x_{3}=\cos \left(\frac{\theta}{2}\right)
$$

$\mathbf{M}_{1}=\left(\begin{array}{lccc}1 & 0 & 0 & 0 \\ X 2 c_{\phi}^{2} c_{\frac{\theta}{2}}^{2}-2 c_{\phi}^{2}-2 c_{\frac{\theta}{2}}^{2}+1 & -2 s_{\frac{\theta}{2}}^{2} s_{\phi} c_{\phi} & 2 s_{\frac{\theta}{2}} s_{\phi} c_{\frac{\theta}{2}} \\ Y & -2 s_{\frac{\theta}{2}}^{2} s_{\phi} c_{\phi} & -2 c_{\phi}^{2} c_{\frac{\theta}{2}}^{2}+2 c_{\phi}^{2}-1 & -2 s_{\frac{\theta}{2}} c_{\phi} c_{\frac{\theta}{2}} \\ Z & 2 s_{\frac{\theta}{2}} s_{\phi} c_{\frac{\theta}{2}} & -2 s_{\frac{\theta}{2}} c_{\phi} c_{\frac{\theta}{2}} & 2 c_{\frac{\theta}{2}}^{2}-1\end{array}\right)$

where

$$
\begin{aligned}
& X=-s_{\frac{\theta}{2}}^{2}\left(2 c_{\phi}^{2}-1\right) \\
& Y=2 s_{\frac{\theta}{2}}^{2} s_{\phi} c_{\phi} \\
& Z=\frac{2 c_{\phi}^{2} c_{\frac{\theta}{2}}^{3}-2 c_{\phi}^{2} c_{\frac{\theta}{2}}-c_{\frac{\theta}{2}}^{3}+c_{\frac{\theta}{2}}-2 y_{2}}{s_{\frac{\theta}{2}} s_{\phi}}
\end{aligned}
$$

where $c_{\phi}=\cos (\phi), s_{\phi}=\sin (\phi), c_{\frac{\theta}{2}}=\cos \left(\frac{\theta}{2}\right)$, and $s_{\frac{\theta}{2}}=\sin \left(\frac{\theta}{2}\right)$.

Figures 2-3 represent the singularity loci of the 3-PRS parallel manipulators for five values of parameter angle $\alpha$, i.e. five designs of the 3-PRS parallel manipulator, at altitude $Z=1 \mathrm{~m}$ and $Z=4 \mathrm{~m}$, respectively. For a given value of azimuth angle $\phi$, the workspace of the 3-PRS parallel manipulator with $\alpha=0^{\circ}$ (the prismatic joints are coplanar to the base) increases with the altitude of the moving platform. Indeed, at the altitude $Z=1 \mathrm{~m}$, the orientation of the moving platform is very limited in which $\theta$ is lower than $15^{\circ}$. When the moving platform reaches the altitude $Z=4 \mathrm{~m}$, the mechanism can tilt up to $60^{\circ}$ for $\phi=30^{\circ}$ without reaching any singularity.

When the prismatic joints of the 3-PRS parallel manipulator are assembled with angle $\alpha=30^{\circ}, \alpha=45^{\circ}$, and $\alpha=60^{\circ}$, the manipulators have larger orientation workspace free of singularity. The workspace of the 3-PRS parallel manipulator with $\alpha=30^{\circ}$ is considerably large and the moving platform can tilt up to $\theta=90^{\circ}$ at $\phi=30^{\circ}, 150^{\circ},-90^{\circ}$ while the manipulator does not reach any singularity. The orientation capability of the 3PRS parallel manipulator with $\alpha=90^{\circ}$ (the prismatic joints are parallel to each other along $\mathbf{z}$-axis) is relatively constant either at lower or at higher altitude.

\section{Orientation Capability in Operation Mode $x_{3}=0$}

The variables $y_{0}, y_{2}, y_{3}$ in this operation mode are solved linearly from the ideal $\mathcal{J}_{2}$. The prismatic lengths $r_{1}, r_{2}, r_{3}$ can are obtained from the equations $g_{4}, g_{5}, g_{6}$ in Eqs. (11-12), respectively. All joint variables and the Study parameters are substituted into the first factor of the determinant of the Jacobian matrix $\mathcal{S}_{2}: \operatorname{det}\left(\mathbf{J}_{2}\right)=0$, hence it is determined in terms of $t_{a}, x_{0}, x_{1}, x_{2}, y_{1}$.

To obtain the orientation of the moving platform, the remaining Study parameters are converted into the tilt and azimuth angles in Eq. (29) and the corresponding transformation matrix $\mathbf{M}_{2}$ is shown in Eq. (30). The altitude of the moving platform $Z$ can be determined as function of parameter $y_{1}$ and tilt and azimuth angles. The design parameters are assigned as $h_{0}=2 \mathrm{~m}, h_{1}=1 \mathrm{~m}$, $l=5 \mathrm{~m}$.

$$
x_{0}=\cos \left(\frac{\theta}{2}\right), \quad x_{1}=\sin \left(\frac{\theta}{2}\right) \cos (\phi), \quad x_{2}=\sin \left(\frac{\theta}{2}\right) \sin (\phi) .
$$

$$
\mathbf{M}_{2}=\left(\begin{array}{cccc}
1 & 0 & 0 & 0 \\
X-2 c_{\phi}^{2} c_{\frac{\theta}{2}}^{2}+2 c_{\phi}^{2}+2 c_{\frac{\theta}{2}}^{2}-1 & 2 s_{\frac{\theta}{2}}^{2} s_{\phi} c_{\phi} & 2 s_{\frac{\theta}{2}} c_{\frac{\theta}{2}} s_{\phi} \\
Y & 2 s_{\frac{\theta}{2}}^{2} s_{\phi} c_{\phi} & 2 c_{\phi}^{2} c_{\frac{\theta}{2}}^{2}-2 c_{\phi}^{2}+1 & -2 s_{\frac{\theta}{2}} c_{\frac{\theta}{2}} c_{\phi} \\
Z & -2 s_{\frac{\theta}{2}} s_{\phi} c_{\frac{\theta}{2}} & 2 s_{\frac{\theta}{2}} c_{\phi} c_{\frac{\theta}{2}} & 2 c_{\frac{\theta}{2}}^{2}-1
\end{array}\right)
$$

where

$$
\begin{aligned}
& X=s_{\frac{\theta}{2}}^{2}\left(2 c_{\phi}^{2}-1\right) \\
& Y=-2 s_{\frac{\theta}{2}}^{2} s_{\phi} c_{\phi} \\
& Z=\frac{2 c_{\phi}^{2} c_{\frac{\theta}{2}}^{3}-2 c_{\phi}^{2} c_{\frac{\theta}{2}}-c_{\frac{\theta}{2}}^{3}+c_{\frac{\theta}{2}}-2 y_{1}}{s_{\frac{\theta}{2}} s_{\phi}}
\end{aligned}
$$

where $c_{\phi}=\cos (\phi), s_{\phi}=\sin (\phi), c_{\frac{\theta}{2}}=\cos \left(\frac{\theta}{2}\right)$, and $s_{\frac{\theta}{2}}=\sin \left(\frac{\theta}{2}\right)$.

The singularity loci of the 3-PRS parallel manipulators for five values of parameter angle $\alpha$, i.e. five designs of the 3-PRS parallel manipulator, at altitude $Z=1 \mathrm{~m}$ and $Z=4 \mathrm{~m}$ are shown in figs. 4-5. In this operation mode, the orientation capability of the 3-PRS parallel manipulators has the same behaviour as in the operation mode $x_{0}=0$. When the prismatic joints are assembled coplanar to the base, the orientation capability of the moving platform is very limited at the altitude $Z=1 \mathrm{~m}$ and it increases significantly at the altitude $Z=4 \mathrm{~m}$. The moving platform can tilt up to $\theta=90^{\circ}$ at $\phi=-30^{\circ}, 90^{\circ},-150^{\circ}$ without being in the singularities.

The higher the altitude $Z$ of the moving platform, the smaller the orientation workspace of the manipulator for $\alpha=30^{\circ}, \alpha=$ $45^{\circ}$, and $\alpha=60^{\circ}$. When the prismatic joints are assembled in 


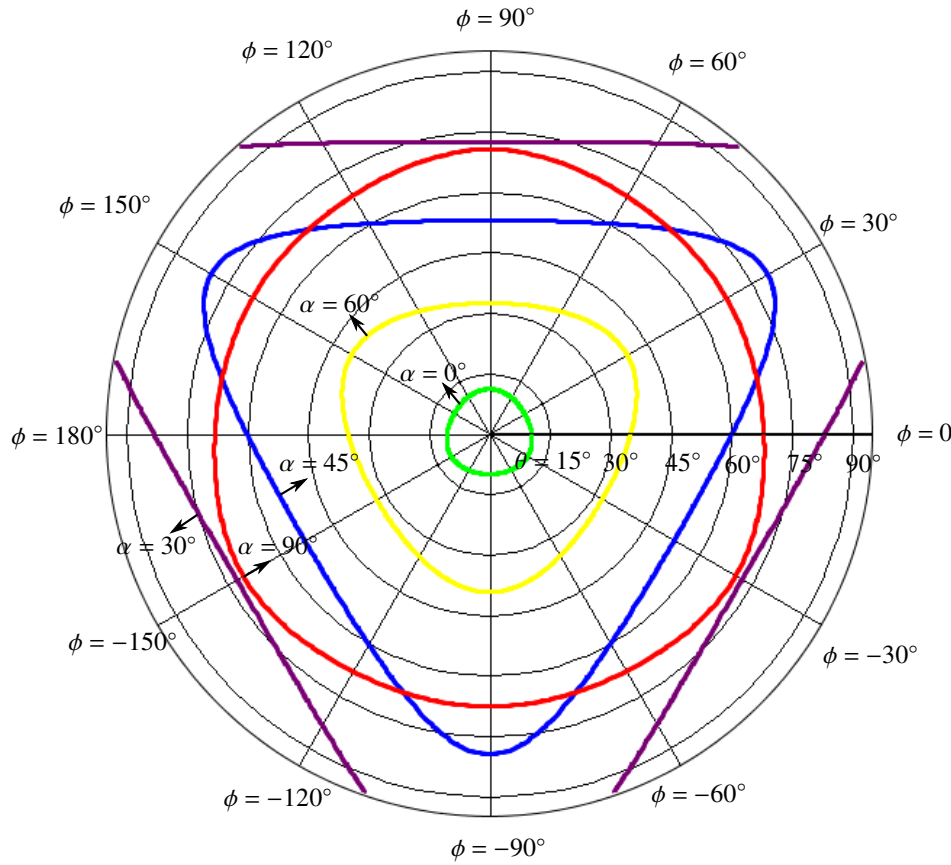

Figure 2: SINGULARITY LOCI IN OPERATION MODE $x_{0}=$ 0, AT $Z=1 \mathrm{~m}$.

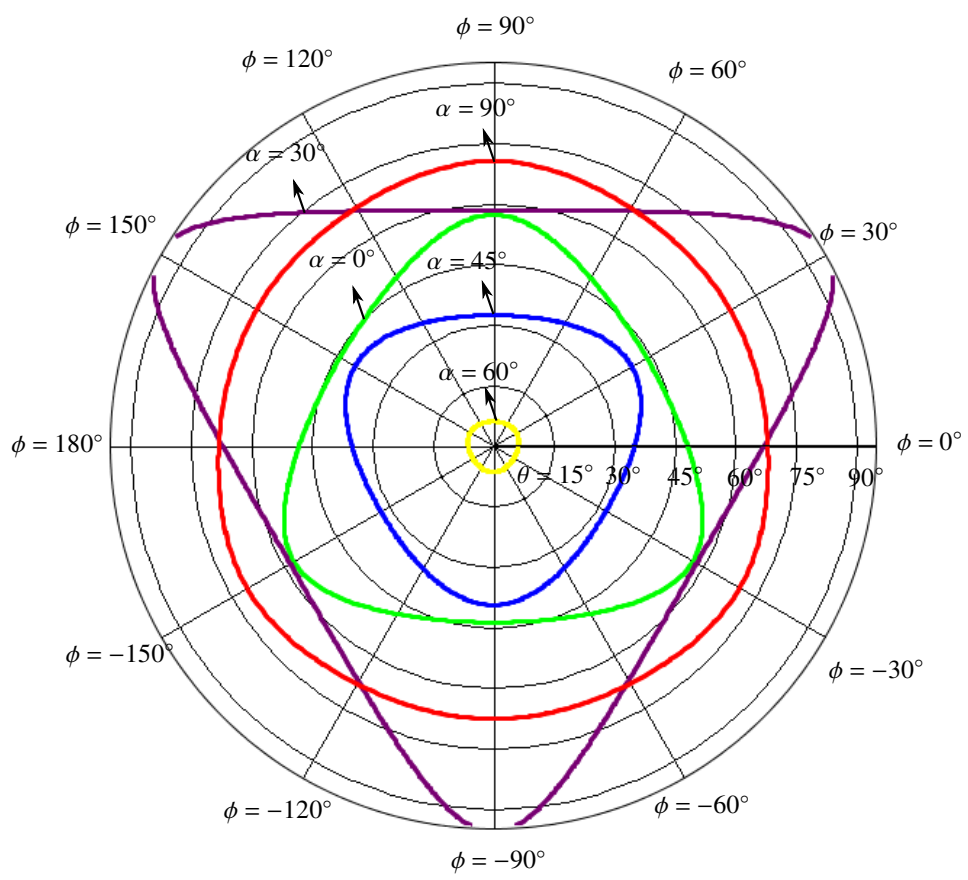

Figure 3: SINGULARITY LOCI IN OPERATION MODE $x_{0}=$ 0, AT $Z=4 \mathrm{~m}$.

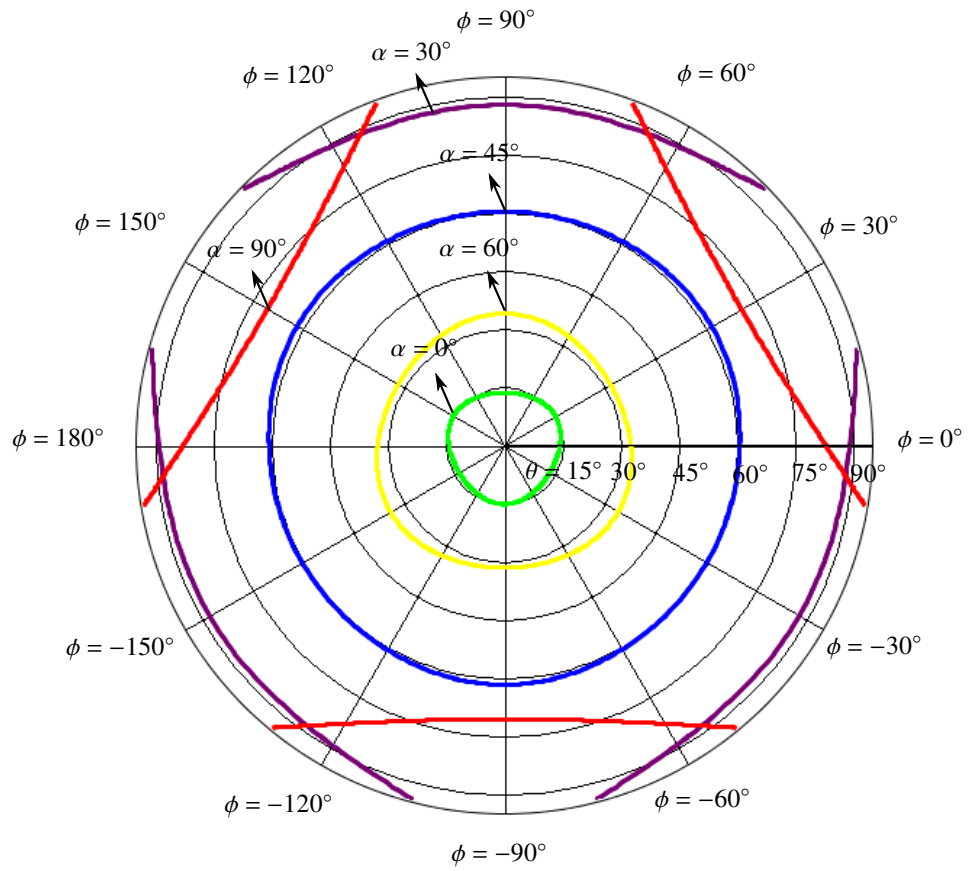

Figure 4: SINGULARITY LOCI IN OPERATION MODE $x_{3}=$ 0 , AT $Z=1 \mathrm{~m}$.

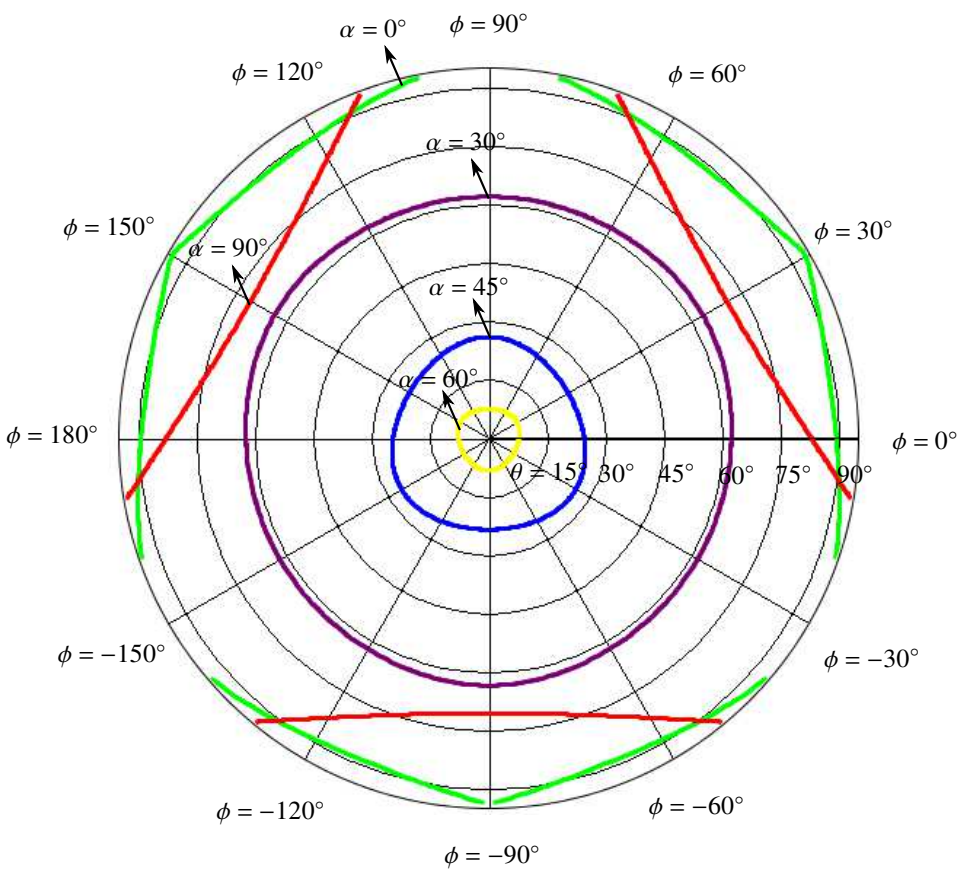

Figure 5: SINGULARITY LOCI IN OPERATION MODE $x_{3}=$ 0, AT $Z=4 \mathrm{~m}$. 
such a way that $\alpha=60^{\circ}$, the moving platform can tilt maximally up to $\pm 7.5^{\circ}$ at the altitude $Z=4 \mathrm{~m}$. For the 3-PRS parallel manipulator with $\alpha=90^{\circ}$, the orientation capability of the moving platform is relatively constant either at lower or at higher altitude. However, its workspace in the operation mode $x_{3}=0$ is bigger than in the operation mode $x_{0}=0$.

\section{TRANSITION OF OPERATION MODES}

The 3-PRS parallel manipulators may switch from one operation mode to another operation mode by passing through a constraint singularity. This singularity lies in the set $x_{0}=x_{3}=0$. From practical point of view, the singularity surface is desirable also in the joint space as a function of angle $\alpha$. The condition $x_{0}=x_{3}=0$ is substituted into one of the systems $\mathcal{K}_{1}$ or $\mathcal{K}_{2}$. Then all Study parameters are eliminated to obtain a single polynomial generated by $r_{1}, r_{2}, r_{3}, t_{a}$.

The design parameters are assigned as $h_{0}=2 \mathrm{~m}, h_{1}=1 \mathrm{~m}$, $l=5 \mathrm{~m}$ and the first prismatic length is assigned as $r_{1}=0$. The Study parameters are eliminated and the computation yields a polynomial of degree 16 in $r_{2}, r_{3}$ and degree 24 in $t_{a}$. Figure 6 represents the singularity surface of the manipulator under study as a function of variables $r_{2}, r_{3}$ and parameter $t_{a}$.

Figure 6 shows that the workspace of the mechanism before changing the operation mode is symmetrical with respect to $t_{a}=0(\alpha=0)$. By taking a point on this surface, we are able to compute the direct kinematics of at least one singularity pose. By selecting five designs of the 3-PRS parallel manipulators, i.e. five different values for angle $\alpha$, the cross sections of the singularity surface in Fig.6 is shown in Fig. 7. It is shown that the largest joint space of the mechanism which is free of operation mode changing, occurs when the prismatic joints are assembled coplanar to the base $(\alpha=0)$. The 3-PRS parallel manipulators in which the prismatic joints are assembled with $\alpha=30^{\circ}$ and $\alpha=60^{\circ}$ will have similar singularity conditions since their curves are coincident.

\section{CONCLUSIONS}

In this paper, an algebraic geometry based approach was applied to analyse the operation modes and the singularities of the 3-PRS parallel manipulator with different arrangements of prismatic joints. A primary decomposition of an ideal of eight constraint equations revealed that the 3-PRS parallel manipulator has always the same number and types of operation modes, namely $x_{0}=0$ and $x_{3}=0$, no matter the angle $\alpha$ between the horizontal base of the manipulator and the directions of the actuated prismatic joints. The interpretation of these operation modes was provided. The singularity conditions were computed. Under the singularity conditions, the orientation capabilities for five different designs of the 3-PRS manipulators were obtained. These orientation capabilities were presented in Euler angles and were

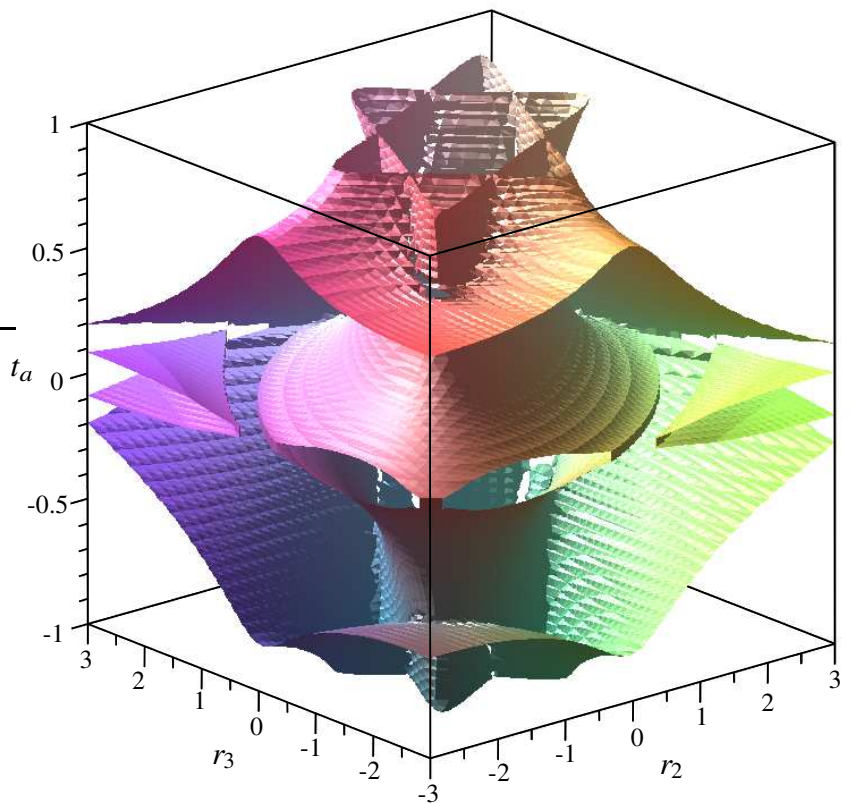

Figure 6: SINGULARITY SURFACE OF $x_{0}=x_{3}=0$.

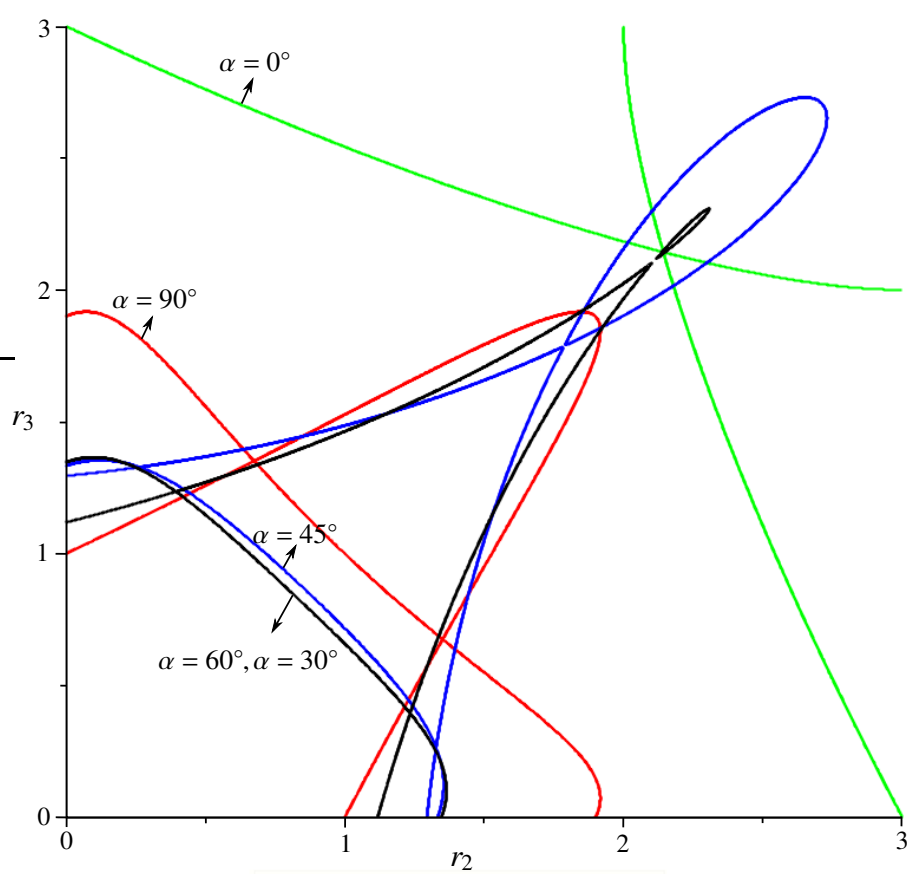

Figure 7: TRANSITION OF OPERATION MODE WITH DIFFERENT ANGLE $\alpha$ 
discussed for both operation modes. It turns out that the when the prismatic joints of the 3-PRS are assembled parallel to each other and vertically $\left(\alpha=90^{\circ}\right)$, the moving platform orientation is relatively constant in both operation modes. When the prismatic joints are coplanar to the base $\left(\alpha=0^{\circ}\right)$, the higher the altitude $Z$ of the moving platform, the larger the orientation workspace of the manipulator free of singularity in both operation modes. For the 3-PRS parallel manipulators with $\alpha=30^{\circ}, \alpha=45^{\circ}$, and $\alpha=60^{\circ}$, the higher the altitude $Z$ of the moving-platform, the smaller the orientation workspace of the manipulator free of singularity in both operation modes. The transition of the operation modes were analysed and represented in the joint space. It turns out that the maximum workspace before changing the operation mode is obtained by with the 3-PRS parallel manipulator with coplanar prismatic joints $\left(\alpha=0^{\circ}\right)$. The investigation of collisions and the analysis of kinematic performances of 3-PRS will be the subject of future work.

\section{REFERENCES}

[1] K.H. Hunt., 1983. "Structural Kinematics of in-parallelactuated Robot-arms". Journal of Mechanisms, Transmissions, and Automation in Design, 105, pp. 705-712.

[2] Wahl, J., 2000. "Articulated Tool Head". WIPO Patent, No. WO 00/25976.

[3] Liu, X-J., and Bonev, I., 2008. "Orientation Capability, Error Analysis, and Dimensional Optimization of Two Articulated Tool Heads With Parallel Kinematics". Journal of Manufacturing Science and Engineering, 130, pp. 011015-1-011015-9.

[4] Liu, X-J., Wang, L-P., Xie, F., and Bonev, I., 2010. ”Desiign of a Three-Axis Articulated Tool Head With Parallel Kinematics Achieving Desired Motion/Force Transmission Characteristics". Journal of Manufacturing Science and Engineering, 132, pp. 021009-1-021009-8.

[5] Tsai, M-S., Shiau, T-N., Tsai, Y-J., and Chang, T-H., 2003. "Direct Kinematics Analysis of a 3-PRS Parallel Mechanism". Mechanism and Machine Theory, 38, pp. 71-83.

[6] Fan, K-C., Wang, Hai., Zhao, J-W., and Chang, T-H., 2003. "Sensitivity analysis of the 3-PRS parallel kinematic spindle platform of a serial-parallel machine tool". International Journal of Machine Tools and Manufacture, 43(15), pp. 1561-1569.

[7] Lin, Qinchuan., Chen, Zhi., Chen, Qiaohong., Wu, Cuanyu., and Hu, Xudong., 2011. "Parasitic motion comparison of 3-PRS parallel mechanism with different limb arrangements". Robotics and Computer-Integrated Manufacturing, 27, pp. 389-396.

[8] Xie, Fugui., Liu, X-J., and Wang, J., 2012. "A 3-DOF parallel manufacturing module and its kinematic optimiza- tion". Robotics and Computer-Integrated Manufacturing, 28, pp. 334-343.

[9] Carbonari, L., Callegari, M., Palmieri, G., and Palpaceli, M.-C., 2014. "A new class of reconfigurable parallel kinematic machines". Mechanism and Machine Theory, 79, pp. 173-183.

[10] Carretero, J.A., Nahon, M.A., Gosselin, C.M., Buckham, B., 1997. "Kinematic Analysis of a Three-DOF Parallel Mechanism for Telescopic Applications". In Proceedings of the ASME Design Automation Conference, Sacramento, California.

[11] Carretero, J.A., Podhorodeski, R.P., Nahon, M.A., and Gosselin, C.M., 2000. "Kinematic Analysis and Optimization of a New Three Degree-of-Freedom Spatial Parallel Manipulator". Journal of Mechanical Design, 122, pp. 1724.

[12] Briot, S., and Bonev, I.A., 2008. "Singularity analysis of zero-torsion parallel mechanisms". Intelligent Robot and Systems, 2008 IROS, IEEE/RSJ International Conference, Nice, pp. 1952-1957.

[13] Li, Yangmin., and $\mathrm{Xu}$, Qingsong., 2007. "Kinematic analysis of a 3-PRS parallel manipulator". Robotics and Computer-Integrated Manufacturing, 23, pp. 395-408.

[14] Nurahmi, L., Schadlbauer, J., Caro, S., Husty, M., and Wenger, P., 2015. "Kinematic Analysis of the 3-RPS Cube Parallel Manipulator". Frontiers of Mechanical Engineering, ASME Journal of Mechanisms and Robotics, 71, pp. 011008-1-011008-10.

[15] Schadlbauer, J., Walter, D.R., and Husty, M., 2014. "The 3-RPS Parallel Manipulator from an Algebraic Viewpoint". Mechanism and Machine Theory, 75, pp. 161-176.

[16] Husty, M.L., Pfurner, M, Schröcker, H-P., and Brunnthaler, K., 2007. "Algebraic Methods in Mechanism Analysis and Synthesis". Robotica, 261, pp. 661-675.

[17] Xianwen Kong., 2014. "Reconfiguration analysis of a 3DOF parallel mechanism using Euler parameter quaternions and algebraic geometry method". Mechanism and Machine Theory, 75, pp. 188-201.

[18] Pottmann, H., and Wallner, J., 2001. Computational Line Geometry. Springer Verlag Berlin.

[19] Schadlbauer, J., Nurahmi, L., Husty, M., Wenger, P., Caro., S, 2013. "Operation Modes in Lower-Mobility Parallel Manipulators". In Second Conference on Interdisciplinary Applications of Kinematics, Lima, Peru, pp. 3-10.

[20] Bottema, O., and Roth, B., 1990. Theoretical Kinematics. Dover Publishing New York.

[21] Lung-Wen Tsai., 1999. Robot Analysis: The Mechanics of Serial and Parallel Manipulators. John Wiley \& Sons Inc.

[22] NASA Mission Planning and Analysis Division., 1977. Euler Angles, Quaternions, and Transformation Matrices. London B Johnson Space Center Houston Texas. 